\title{
Bone marrow mesenchymal stem cell-derived exosomal miR-144-5p improves rat ovarian function after chemotherapy-induced ovarian failure by targeting PTEN
}

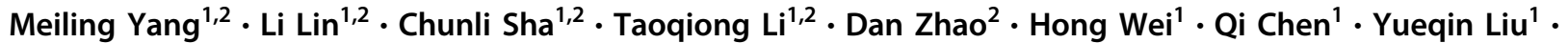 \\ Xiaofang Chen ${ }^{2} \cdot$ Wenlin $\mathrm{Xu}^{1,2} \cdot$ Yuefeng $\mathrm{Li}^{3} \cdot$ Xiaolan $\mathrm{Zhu}^{1,2}$
}

Received: 17 June 2019 / Revised: 11 August 2019 / Accepted: 12 August 2019 / Published online: 19 September 2019

(c) The Author(s), under exclusive licence to United States and Canadian Academy of Pathology 2019

\begin{abstract}
Chemotherapy-induced premature ovarian failure (POF) in women is currently clinically irreversible. Bone marrow mesenchymal stem cells (BMSCs) are a promising cellular therapeutic strategy for POF. However, the underlying mechanism governing the efficacy of BMSCs in treating POF has not been determined. In this study, we show that BMSC and BMSC-derived exosome transplantation can significantly recover the estrus cycle, increase the number of basal and sinus follicles in POF rats, increase estradiol $\left(\mathrm{E}_{2}\right)$ and anti-Mullerian hormone $(\mathrm{AMH})$ levels, and reduce follicle stimulating hormone (FSH) and luteinizing hormone ( $\mathrm{LH}$ ) levels in the serum. Furthermore, we demonstrate that BMSC-derived exosomes prevent ovarian follicular atresia in cyclophosphamide (CTX)-treated rats via the delivery of miR-144-5p, which can be transferred to cocultured CTX-damaged granulosa cells (GCs) to decrease GC apoptosis. A functional assay revealed that overexpression of miR-144-5p in BMSCs showed efficacy against CTX-induced POF, and the improvement in the repair was related to the inhibition of GC apoptosis by targeting PTEN. The opposite effect was exhibited when miR-144-5p was inhibited. Taken together, our experimental results provide new information regarding the potential of using exosomal miR-144-5p to treat ovarian failure.
\end{abstract}

These authors contributed equally: Meiling Yang, Li Lin, Chunli Sha, Taoqiong Li

Supplementary information The online version of this article (https:// doi.org/10.1038/s41374-019-0321-y) contains supplementary material, which is available to authorized users.

Wenlin $\mathrm{Xu}$

xw1630806@126.com

$\triangle$ Yuefeng Li

liyuefeng@ujs.edu.cn

$\triangle$ Xiaolan Zhu

zx12517@163.com

1 Reproductive Sciences Institute, Jiangsu University, Zhenjiang 212003 Jiangsu, China

2 Department of Gynecologic Oncology and Central Laboratory, Fourth Affiliated Hospital of Jiangsu University, Zhenjiang 212001 Jiangsu, China

3 Department of Oncology, Affiliated People Hospital of Jiangsu University, Zhenjiang 212001 Jiangsu, China

\section{Introduction}

Premature ovarian failure (POF) is diagnosed by the following criteria: woman aged $<40$ years with amenorrhea lasting longer than 4 months, follicle-stimulating hormone (FSH) levels $>40 \mathrm{U} / \mathrm{L}$ in at least two measurements and estradiol $\left(\mathrm{E}_{2}\right)$ concentration $<73.2 \mathrm{pmol} / \mathrm{L}$ [1]. The incidence of POF is increasing, and the causes of POF include chromosomal abnormalities, autoimmune factors, environmental factors, psychological factors, and iatrogenic factors. Iatrogenic injuries are common in patients undergoing chemotherapy, radiotherapy, and ovariectomy [2]. Because of the young age at onset in patients suffering from some cancers [3], and the increased long-term survival rate after surgery, more attention has been devoted to treating the reproductive injury by chemotherapy and radiotherapy [4]. The senescence and apoptosis of granulosa cells (GCs) are important causes of decreased ovarian reserve [5], and follicular atresia triggered by excessive loss of GCs plays a role in POF [6].

Recently, stem cell-mediated repair has been described for neuron repair [7], wound healing [8], acute myocardial 
infarction [9], and liver regeneration [10], and has the potential for broad application in the repair of POF [11-13]. Bone marrow-derived mesenchymal stem cells (BMSCs) feature advantages such as self-replication, multidirectional differentiation, convenient source, ability to avoid immune rejection, and lack of ethical issues [14]. However, BMSCs present the following disadvantages: invasive operation, uncontrollable quality in preparation, possible immune response to allograft transplantation, and potential tumorigenicity [15]. Therefore, a treatment that can maintain the repair properties of BMSCs and avoid these disadvantages is urgently needed.

Exosomes, vesicle-like bodies with a diameter of $40-100 \mathrm{~nm}$ secreted by cells, carry a variety of proteins, mRNAs and microRNAs (miRNAs), and participate in cell communication and migration, angiogenesis, and tumor cell growth [16]. Exosomes have stable biological characteristics and lipid bilayer membranes, which can prevent content degradation and allow their direct entry into target cells. This "carrier" signal transduction mode is efficient and stable. Moreover, studies have shown that exosomes derived from BMSCs can also participate in tissue repair and are expected to replace stem cells as a new therapeutic tool for tissue repair [17].

Exosome-encapsulated miRNAs are stable endogenous noncoding miRNAs that bind to partially complementary mRNA sequences, resulting in target degradation or translation inhibition $[18,19]$. Previous studies have shown that miRNAs are differentially expressed in the plasma of patients with POF and normal individuals and play important roles in the pathogenesis, early diagnosis, and evaluation of the therapeutic efficacy of POF [20]. Among miRNAs, miRNA-144 is downregulated in the plasma of POF patients $[21,22]$. Furthermore, miRNA-144-5p is downregulated in the POF tissues of animal models compared with that in normal ovarian tissues [23, 24], although its role in and underlying mechanism of POF regulation remains to be further clarified.

In this study, we investigated the potential therapeutic efficiency of BMSCs in POF rats and the underlying mechanism and found that BMSC-derived exosomal miR144-5p inhibited the apoptosis of damaged GCs and improved ovarian function in rats following cyclophosphamide (CTX) treatment. Exogenous expression of miR144-5p in CTX-induced rats effectively relieved follicles from atresia and repressed apoptosis in GCs in vivo and in vitro, while inhibition of miR-144-5p in BMSCs attenuated the antiapoptotic effect on CTX-damaged GCs in vitro. Our study indicates the therapeutic potential of BMSC-derived exosomal miR-144-5p in POF and helps elucidate a cell-free therapeutic strategy for POF treatment.

\section{Materials and methods}

\section{Experimental animals}

Female clean-grade Sprague Dawley (SD) rats weighing $\sim 150 \mathrm{~g}$ were provided by the laboratory animal center of Jiangsu University. The rats were housed at room temperature $\left(25 \pm 2{ }^{\circ} \mathrm{C}\right)$ with a humidity of $45-55 \%$ and a light duration of $12 \mathrm{~h}$. All experimental procedures involving rats were approved by the Institutional Animal Care and Use Committee of Jiangsu University.

\section{Establishment of the POF rat model}

Five-week-old SD female rats were intraperitoneally injected with $50 \mathrm{mg} / \mathrm{kg}$ CTX (Sigma-Aldrich, St. Louis, MO, USA) on the first day and then with $8 \mathrm{mg} / \mathrm{kg} / \mathrm{d}$ CTX consecutively for 14 days as described in the literature [25].

\section{Estrous cycle examination}

Vaginal cells were collected using a sterile cotton swab, smeared on glass microscope slides, and stained with crystal violet. The morphologies of cells in each smear were observed under a microscope at $\times 200$ magnification, and the estrous cycle of SD rats was distinguished according to the cell type [26].

\section{Enzyme-linked immunosorbent assay}

At the end of the study, blood samples were obtained from eyeball veins and centrifuged at $3220 \times g$ for $15 \mathrm{~min}$. FSH, estradiol $\left(\mathrm{E}_{2}\right)$, anti-Mullerian hormone $(\mathrm{AMH})$, and luteinizing hormone (LH) levels in the serum were measured using ELISA kits (Lengton, Shanghai, China) according to the manufacturer's instructions.

\section{Isolation and culture of BMSCs}

BMSCs were isolated from the tibias and femurs of 5-weekold healthy SD rats. The bone marrow was cultured in lowglucose Dulbecco's modified Eagle's medium (LDMEM) supplemented with $20 \%$ fetal bovine serum (Thermo Fisher, Carlsbad, CA), $100 \mathrm{U} / \mathrm{mL}$ penicillin, and $100 \mu \mathrm{g} / \mathrm{mL}$ streptomycin. The cells were seeded into culture vessels at a density of $1 \times 10^{6}$ cells $/ \mathrm{mL}$ and cultured in a humidified incubator at $37{ }^{\circ} \mathrm{C}$ with $5 \% \mathrm{CO}_{2}$. The medium was replaced after $48 \mathrm{~h}$ of culture, and adherent cells were recognized as BMSCs. BMSCs passaged three to seven times with a uniform morphological appearance as fibroblast-like long spindles in an ordered arrangement were used in all experiments. 
BMSC surface marker expression was analyzed by an FACS Calibur flow cytometer (BD Biosciences, San Jose, CA) using CD19-FITC, CD45-FITC, CD34-FITC, CD105PE, and CD90-PE (all from Biolegend, San Diego, California).

The multipotential differentiation of the BMSCs was determined for osteogenesis, which was induced by culturing BMSCs in osteogenic induction medium (Cyagen, Santa Clara, USA) for 14-21 days; calcium deposition was authenticated by Alizarin red staining.

\section{Exosome collection, characterization, quantification, labeling, and tracking}

BMSCs at $\sim 80 \%$ confluence were cultured for $48 \mathrm{~h}$, and the collected supernatant was centrifuged at increasing speeds of $300 \times g$ for $10 \mathrm{~min}, 2000 \times g$ for $10 \mathrm{~min}$, and $10,000 \times g$ at $4{ }^{\circ} \mathrm{C}$ for $30 \mathrm{~min}$ to remove sediment and debris and thus obtain pure exosomes. The supernatant was centrifuged at $100,000 \times g$ for $2 \mathrm{~h}$ to pellet the exosomes in clear ultracentrifuge tubes (Beckman Coulter, Indianapolis, USA). Then, the isolated exosomes were resuspended in $100 \mu \mathrm{L}$ of particle-free PBS. To confirm the successful isolation of exosomes, western blotting was performed to detect the exosome marker proteins CD9, CD81, and tubulin (all from Abcam, Cambridge, UK). Transmission electron microscopy (TEM) was performed to verify the presence of exosomes. Exosomes were dissolved in PBS buffer, dropped onto a carbon-coated copper grid, and then stained with $2 \%$ uranyl acetate. Images of exosomes were obtained using an HT 7Tecnai G2 Spirit electron microscope (FEI Co, Oregon, USA). The exosomes from BMSCs were labeled with PKH67 (Sigma-Aldrich, St. Louis, MO, USA) according to the manufacturer's instructions. The uptake of labeled exosomes by GCs was detected by a Leica TCS SP5 II laser scanning confocal microscope.

\section{BMSC transplantation and exosome injection}

We randomly divided the rats into five equal groups with ten rats in each group. The WT group consisted of normal control rats that received no treatment. In the POF group, the rats were administered CTX. In the PBS group, POF rats were injected intraperitoneally with $100 \mu \mathrm{L}$ of PBS every other day for 2 weeks. In the BMSC group, POF rats were injected intraperitoneally with $1 \times 10^{6} \mathrm{BMSCs}$ in a $100 \mu \mathrm{l}$ volume of PBS every other day for 2 weeks. In the EXO group, POF rats were injected intraperitoneally with BMSC-derived exosomes; specifically, $150 \mu \mathrm{g}$ of exosomal proteins (approximate amount produced by $1 \times 10^{6} \mathrm{BMSCs}$ overnight) in $100 \mu \mathrm{L}$ of PBS was injected every other day for 2 weeks.

\section{Ovarian morphological and follicle counts}

Ovaries were fixed with $4 \%$ paraformaldehyde (PFA) at $4{ }^{\circ} \mathrm{C}$ overnight, embedded in paraffin, serially sectioned $(8 \mu \mathrm{m})$, and stained with hematoxylin-eosin. The ovarian histological examination was performed using light microscopy. The different categories of follicles were determined as previously described [27]. Only follicles containing an oocyte with a clearly visible nucleus were scored.

\section{Isolation and culture of GCs}

Gonadotrophin from pregnant mare serum (PMSG, 20 IU, Sigma-Aldrich, St. Louis, MO, USA) was injected intraperitoneally into 5-week-old SD female rats. After $48 \mathrm{~h}$, the rats were euthanized, and their ovaries were isolated mechanically. GCs were obtained by puncturing the preovulatory follicles under a stereomicroscope. GCs were cultured in high-glucose DMEM (HDMEM) supplemented with $10 \%$ fetal bovine serum (Thermo Fisher, Carlsbad, CA), $100 \mathrm{U} / \mathrm{mL}$ penicillin, and $100 \mu \mathrm{g} / \mathrm{mL}$ streptomycin. Cells were incubated at $37^{\circ} \mathrm{C}$ in a humidified atmosphere of $5 \% \mathrm{CO}_{2}$. The GCs were incubated with the folliclestimulating hormone receptor (FSHR) (Abcam, Cambridge, $\mathrm{UK}$ ) primary antibody for $12 \mathrm{~h}$ at $4{ }^{\circ} \mathrm{C}$ in a humidity chamber and then with the $\mathrm{Cy} 3$-conjugated secondary antibody (Abcam, Cambridge, UK) for $30 \mathrm{~min}$ at $37^{\circ} \mathrm{C}$. After staining the nucleus with DAPI, the signals from each sample were examined by fluorescence microscopy.

\section{Induction of GC apoptosis in vitro and coculture of GCs and BMSCs}

In total, $1 \times 10^{6} \mathrm{GCs}$ were seeded into the lower chambers of six-well plates. CTX was added to the GC culture medium at $2 \mu \mathrm{M}$ to induce apoptosis. After exposure to CTX for $24 \mathrm{~h}$, the GCs were cocultured with BMSCs in the Transwell system. Approximately $5 \times 10^{5}$ BMSCs were seeded into the upper chambers of six-well hanging cell culture inserts (Merck Millipore, Darmstadt, Germany). Because the pore size was $0.4 \mu \mathrm{m}$, the GCs and BMSCs shared the same medium but were kept separate.

\section{Reverse transcription quantitative real-time PCR}

Total RNA was extracted using TRIzol reagent (Invitrogen Corp, Carlsbad, California) according to the manufacturer's protocol. Subsequently, complementary DNA (cDNA) was synthesized using the FastQuant RT Kit (with gDNase, Tiangen, Beijing, China). Real-time polymerase chain reaction (qRT-PCR) analyses were performed using a miRcute Plus miRNA qPCR Detection Kit (Tiangen, Beijing, China). 
The miRNA and mRNA expression levels were analyzed by the $2^{-\Delta \Delta C T}$ method and normalized to those of U6 snRNA and $\beta$-actin, respectively. The primer sequences were $5^{\prime}$ CGCGGGATATCATATACTGTAAGT-3' for miR-144-5p and 5'-TGGATTCGACTTAGACTTGACCT-3' and 5'-TG GGGTGTCATAATGTCTTTC-3' for PTEN.

\section{Transfection}

BMSCs seeded into six-well plates $\left(5 \times 10^{5}\right.$ cells/well $)$ were transfected with miR-144-5p agomir, antagomir or the corresponding negative control (agomir-NC, antagomirNC) (GenePharma, Shanghai, China) using Lipofectamine ${ }^{\mathrm{TM}}$ 2000 (Invitrogen, Carlsbad, CA) according to the manufacturer's instructions. The transfection efficiency was assessed by qRT-PCR at $24 \mathrm{~h}$ after transfection.

\section{Western blot analysis}

Proteins from cells and ovarian tissues were separated by sodium dodecyl sulfate-polyacrylamide gel electrophoresis and transferred onto polyvinylidene difluoride membranes (Bio-Rad Laboratories, Bio-Oxford, USA). After blocking, the membranes were immunoblotted with PTEN, p-AKT, and $\beta$-actin (all from Abcam, Cambridge, UK) primary antibodies and then with horseradish peroxidase-conjugated secondary antibodies.

\section{Evaluation of apoptosis}

An In situ Cell Death Detection Kit, Fluorescein (Roche, Germany), was used to detect GC apoptosis in rat ovarian tissues according to the manufacturer's instructions. The sections were observed with a fluorescence microscope. The apoptotic GCs in the ovarian tissue were stained green, and five nonrepeating regions (ten samples/group) were selected for counting in each sample. GCs were double-stained with Annexin-V-FITC and propidium iodide to evaluate apoptosis using a cell apoptosis kit (BD Biosciences, San Jose, CA).

\section{Immunohistochemistry}

Bilateral ovaries were fixed in a 4\% PFA solution and embedded in paraffin wax. The ovarian tissues were sectioned at a $4 \mu \mathrm{m}$ thickness, dewaxed in gradient alcohol and xylene, and incubated with the primary antibodies caspase 3, caspase 9, and PTEN (all from Abcam, Cambridge, UK) for $12 \mathrm{~h}$ at $4{ }^{\circ} \mathrm{C}$ in a humid environment. The sections were then incubated with a biotinylated secondary antibody for $1 \mathrm{~h}$ at $37^{\circ} \mathrm{C}$. Five sections on each slide were randomly selected for examination. German immunoreactive score (IRS) criteria were used to score the staining results. Briefly, the staining signal intensity was graded as " 0 " (negative),
"1" (weak), "2" (moderate), or "3" (strong); the extent of staining cells was graded as " 0 " $(<5 \%)$, "1" $(5-25 \%)$, " 2 " $(25-50 \%)$, "3" $(50-75 \%)$ or " 4 " $(>75 \%)$. The values of staining intensity and staining extent were multiplied to obtain the final IRS [28].

\section{Statistical analysis}

At least three biological replicates were performed for every experiment in this study, and all values are presented as the mean \pm standard error of the mean. An unpaired $t$ test and ANOVA with Tukey's multiple comparison test were used to analyze the data among groups. $P<0.05$ was defined as statistically significant.

\section{Results}

\section{Cultivation and characterization of rat BMSCs and BMSC-derived exosomes}

BMSCs passaged three to seven times had a uniform morphologica appearance as fibroblast-like long spindles in an ordered arrangement (Fig. 1a). BMSCs possessing a multilineage differentiation capability were determined based on their ability to undergo osteogenesis (Fig. 1b). Flow cytometry was used to identify the expression of surface markers, and over $90 \%$ of BMSCs were negative for CD34, CD19, and CD45 and positive for CD90 and CD105 (Fig. 1c). The characteristics of BMSC-derived exosomes were analyzed by electron microscopy, which revealed vesicular structures (Fig. 1d). Western blot, the universally recognized method for identifying circulating exosomes, was used to assess the expression of the exosomal marker proteins CD9 and CD81 (Fig. 1e).

\section{Therapeutic effects of BMSCs and BMSC-secreted exosomes on POF rats}

To explore the roles of BMSCs and BMSC-secreted exosomes in POF rats, we first established a POF rat model and then treated these rats with PBS, BMSCs, and BMSCderived exosomes. The estrous cycles of rats in each group were examined over a 4-week period. Regular estrous cycles were shown in the WT group and lasted 4-6 days, which included proestrus for 1 day, estrus for 1-2 days, metestrus for 1 day, and diestrus for 1-2 days (Fig. 2a1-4). However, significantly irregular estrous cycles were observed in POF rats as shown in Fig. 2b, c. After BMSC or BMSC-derived exosome transplantation for 2 weeks, the number of rats with normal estrous cycles increased. These results suggested that BMSCs or BMSC-derived exosomes could restore the estrus cycle of POF rats. The FSH and LH 


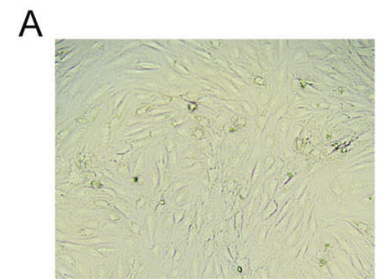

B

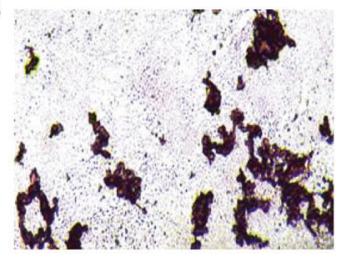

c
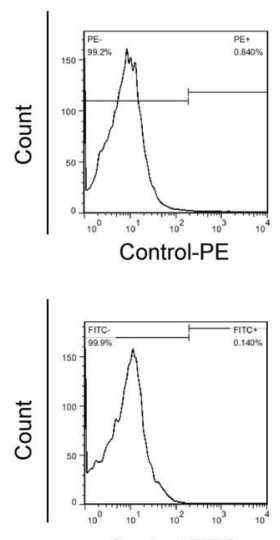

Control-FITC

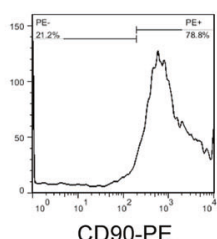

CD90-PE

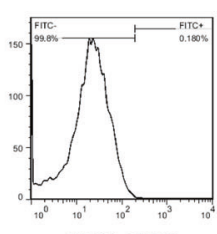

CD34-FITC
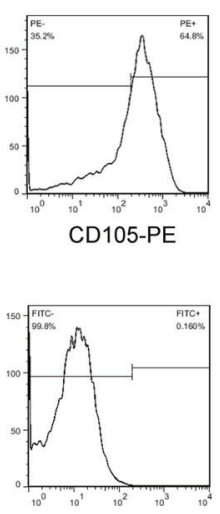

CD45-FITC
D
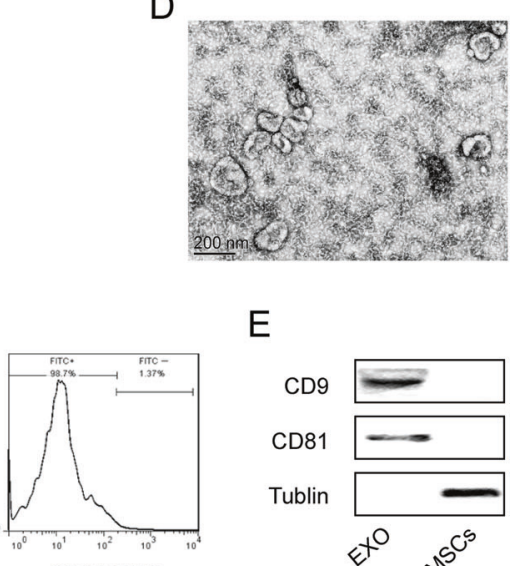

CD19-FITC
$\mathrm{E}$

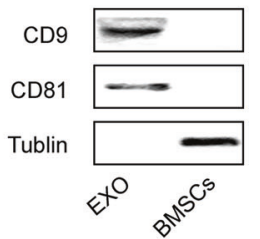

Fig. 1 Isolation and identification of BMSCs and BMSC-derived exosomes. a Third generation BMSCs exhibited a typical fibroblastic morphology. Scale bar: $100 \mu \mathrm{m}$. b BMSCs differentiated into osteoblasts. Scale bar: $100 \mu \mathrm{m}$. c FCM analysis of BMSCs. The cells were positive for $\mathrm{CD} 90$ and $\mathrm{CD} 105$ and negative for CD34, CD45, and
CD19. d Electron microscopy image of exosomes isolated from BMSC-conditioned medium. Scale bar: $200 \mathrm{~nm}$. e Western blot analysis of CD9, CD81, and tubulin expression in BMSC-derived exosomes (EXO) and BMSCs

PBS group, the caspase 3 and caspase 9 levels in the BMSC and EXO groups were decreased $(P<0.001)$ (Supplementary Fig. 1C, D). According to the above results, BMSCs or BMSC-derived exosomes relieved CTX-induced ovarian tissue injury. These results confirmed that BMSCs and BMSC-derived exosomes could improve ovarian function and inhibit GC apoptosis.

\section{BMSC-derived exosomes contributed to the antiapoptotic effect on damaged GCs}

Morphological analysis of the GCs revealed polygonal and fibrous-like structures (Fig. 3a). After immunofluorescence analysis of FSHR, GCs were stained red with $\mathrm{Cy} 3$, and $\sim 90 \%$ of the adherent cells were positively stained (Fig. 3b), indicating that GCs were successfully cultured in vitro.

BMSC-derived exosomes labeled with PKH67 were cocultured with CTX-damaged GCs. The exosomes were incorporated into the cytoplasm of CTX-damaged GCs (Fig. 3c), which verified that BMSC-derived exosomes were taken up by CTX-damaged GCs.

To further clarify the effects of BMSCs and BMSCderived exosomes on CTX-damaged GCs, we cocultured CTX-damaged GCs with BMSCs, BMSCs pretreated with GW4689, PBS or BMSC-derived exosomes. After staining the GCs in each group with annexin V-FITC and PI, the apoptotic GCs were counted by flow cytometry (Fig. 3d). The apoptosis rate of CTX-damaged GCs cocultured with BMSCs or BMSC-derived exosomes was decreased (Fig. 3e). These results suggest that BMSCs or exosomes inhibit the apoptosis of CTX-damaged GCs in vitro. However, when GCs were cocultured with BMSCs pretreated with GW4869, which prevented the release of exosomes in 
A

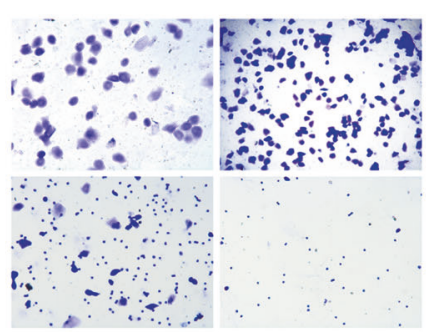

B

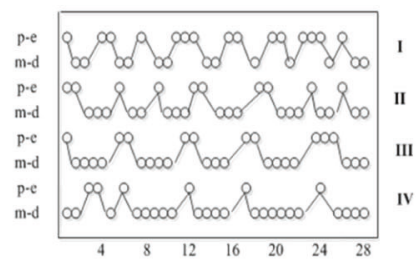

C

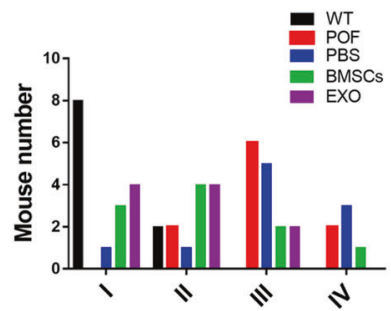

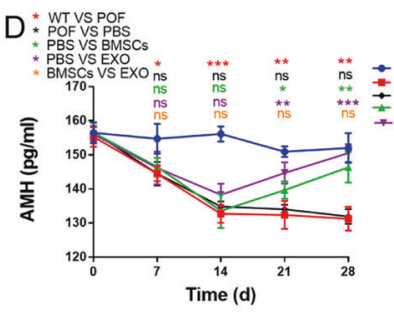

$E$
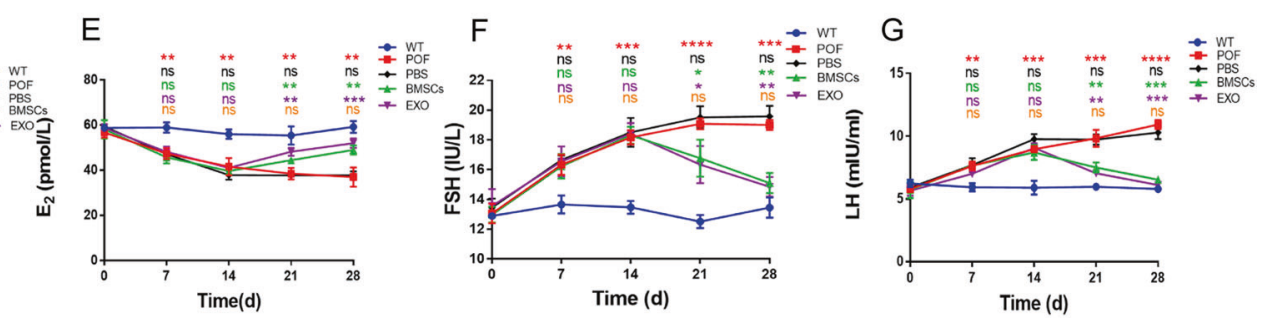

$\mathrm{H}$
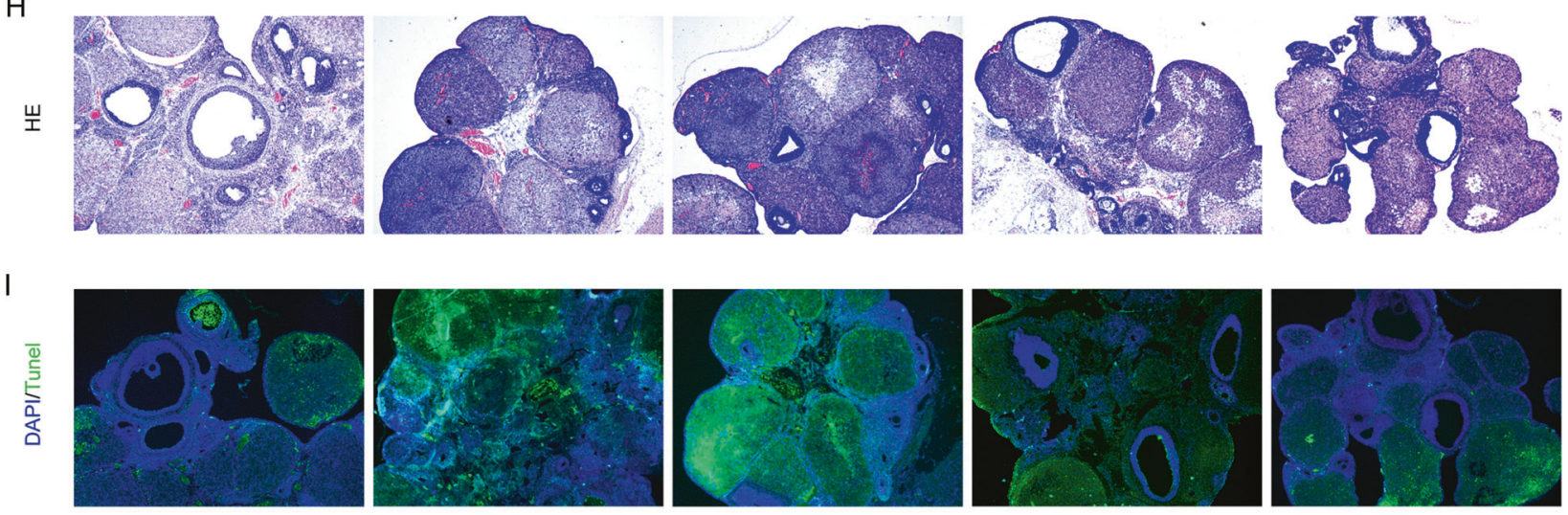

$J$
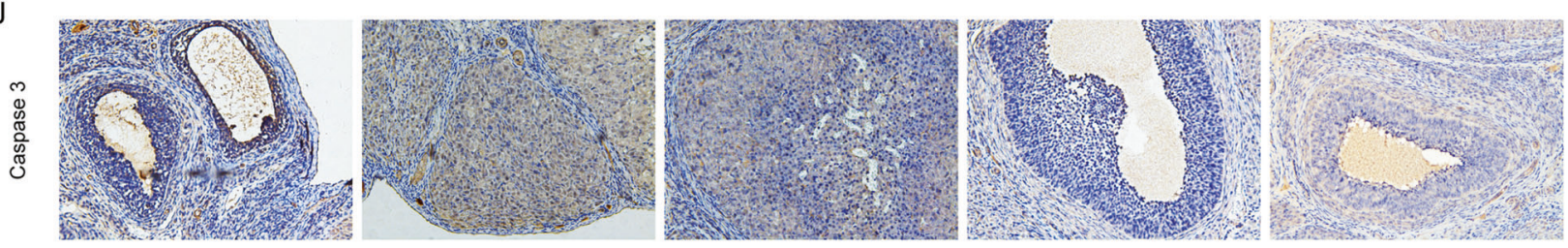

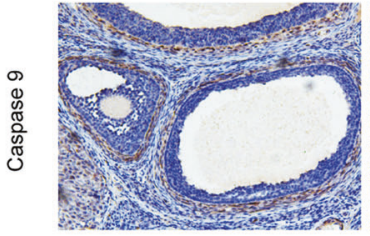

WT

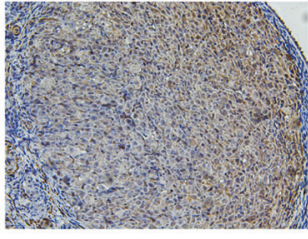

POF

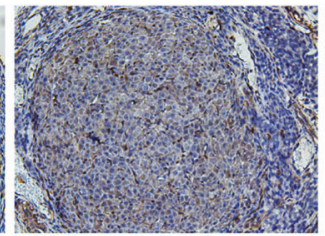

PBS

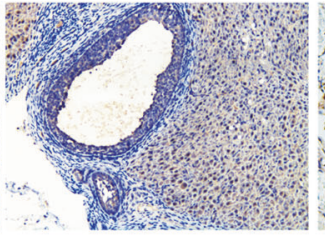

BMSC

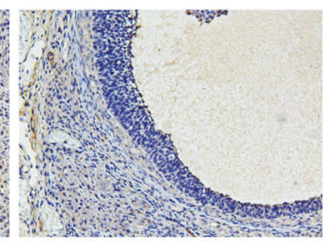

EXO
Fig. 2 Therapeutic effects of BMSCs and BMSC-secreted exosomes on POF rats. a Normal estrous cycles: 1, proestrus; 2, estrus; 3, metestrus; 4, diestrus. Scale bar: $100 \mu \mathrm{m}$. b Four patterns of estrous cycles were graded based on the severity of abnormality (I-IV): I, normal; II, regular cycles with shortened estrus; III, irregular cycles with prolonged diestrus and normal or prolonged estrus; IV, no cyclicity. The $y$ axis represents the cycle day in proestrus or estrus $(\mathrm{p}-\mathrm{e})$ and metestrus or diestrus $(\mathrm{m}-\mathrm{d})$. Each circle $(\mathrm{O})$ represents one rat. The illustration represents only one rat and is meant to show the change in estrus cycle. c Total numbers of rats from each group categorized into the various estrous patterns (I-IV). Trends for the average levels of AMH (d), $\mathrm{E}_{2}(\mathbf{e}), \mathrm{FSH}(\mathbf{f})$ and $\mathrm{LH}(\mathbf{g})$. Comparison of the $\mathrm{AMH}, \mathrm{E}_{2}, \mathrm{FSH}$, and LH levels between groups (*POF vs. WT, *POF vs. PBS, *PBS vs. BMSCs, *PBS vs. EXO, *BMSCs vs. EXO) at different times. The "ns" $(P>0.05)$ indicates no statistically significant difference. $* P<0.05$, $* * P<0.01, * * * P<0.001$, $* * * * P<$ 0.0001 for all figures. h Hematoxylin and eosin staining. Scale bar: $50 \mu \mathrm{m}$. i TUNEL assay of GC apoptosis. The green stain indicates tunnel-positive GCs. The blue stain indicates the nucleus. Scale bar: $50 \mu \mathrm{m}$. $\mathbf{j}$ The expression of caspase 3 and caspase 9 in ovarian tissues as determined by immunochemistry analysis. Scale bar: $200 \mu \mathrm{m}$ 
A

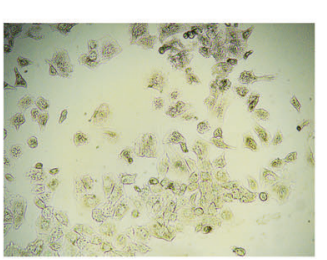

B

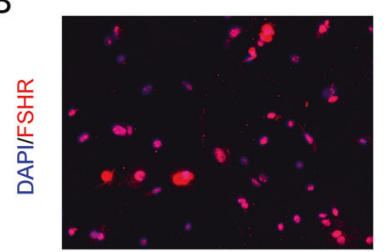

C

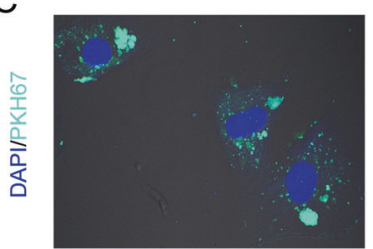

D

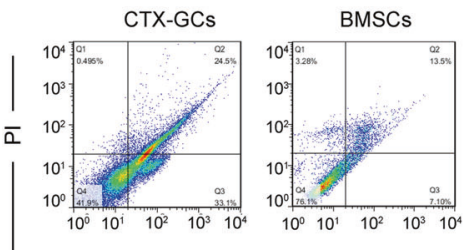

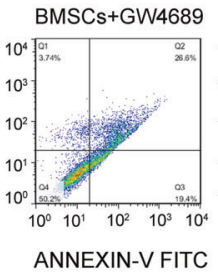

PBS

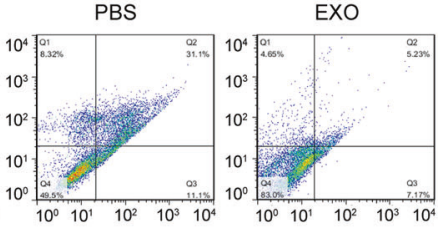

$\mathrm{E}$

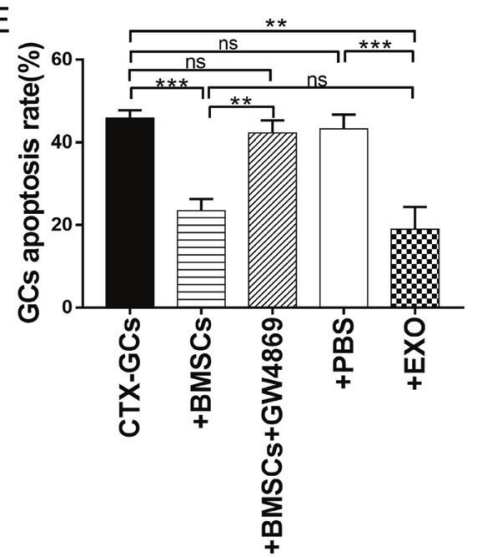

Fig. 3 BMSC-derived exosomes could inhibit the apoptosis of GCs. a Morphology of GCs. Scale bar: $100 \mu \mathrm{m}$ b FSHR (red) expression in GCs. Nuclei were stained with DAPI (blue). Scale bar: $100 \mu \mathrm{m}$. c PKH67-labeled BMSC-derived exosomes (green) were incorporated

BMSCs, the apoptosis rate of GCs did not decrease. These results indicate that BMSCs inhibit the apoptosis of CTXdamaged GCs mainly via exosomal transfer.

\section{Therapeutic effects of exosomal miR-144-5p derived from BMSCs on damaged GCs}

Recently, miRNAs have been identified in exosomes, which can be taken up by neighboring or distant cells and subsequently modulate recipient cells [29]. A previous study profiled differentially expressed miRNAs associated with POF development. Among miRNAs, miR144-5p was downregulated in rat POF tissues compared with that in control tissues [23]. To validate that BMSCs could deliver miR-144-5p via exosomes to inhibit GC apoptosis, the expression levels of mir-144-5p in GCs were detected by qRT-PCR (Fig. 4a). miR-144-5p expression was increased in CTX-damaged GCs cocultured with BMSCs or exosomes. When CTX-damaged GCs were cocultured with BMSCs pretreated with GW4869, miR-144-5p expression remained unchanged. The expression of miR-144-5p inhibited the apoptosis of GCs, indicating that BMSCderived exosomal miR-144-5p had a protective effect against the apoptosis of CTX-damaged GCs.

We next examined whether miR-144-5p contributed to the therapeutic effect. We overexpressed or inhibited miR144-5p in BMSCs or BMSC-derived exosomes using into the cytoplasms of damaged GCs. Nuclei were stained with DAPI (blue). Scale bar: $20 \mu \mathrm{m}$. d The apoptosis rates of GCs as determined by flow cytometry. e Comparison of the percentages of apoptotic GCs in each group

agomir and antagomir, respectively, and then confirmed overexpression or inhibition by qRT-PCR (Fig. 4b). After the transfection of agomir and antagomir into exosomes, CTX-damaged GCs were cocultured with the exosomes. As shown in Fig. 4c-e, miR-144-5p overexpression inhibited the chemotherapy-induced apoptosis of GCs. Consistent with these findings, decreasing miR-144-5p expression increased the apoptosis rate of GCs. In summary, the exosomal miR-144-5p derived from BMSCs exerted a therapeutic effect on CTX-damaged GCs.

\section{Exosomal miR-144-5p inactivated the PI3K/AKT pathway by suppressing PTEN targeting}

To further understand the mechanism by which BMSC exosomal miR-144-5p inhibited the apoptosis of GCs, the expression of PTEN in ovarian tissue was detected by immunohistochemistry and western blot analysis (Fig. 5a, b). PTEN expression was increased in the POF group compared with that in the WT group. Compared with those in the PBS group, the follicles observed in the BMSC and EXO groups were healthier, and significantly decreased PTEN expression was observed (Supplementary Fig. 2). Next, we verified that PTEN expression was downregulated in CTX-damaged GCs cocultured with BMSCs or exosomes (Fig. 5c). MiR-144-5p inhibits CTX-damaged GC apoptosis, likely through regulation of the PI3K/Akt 


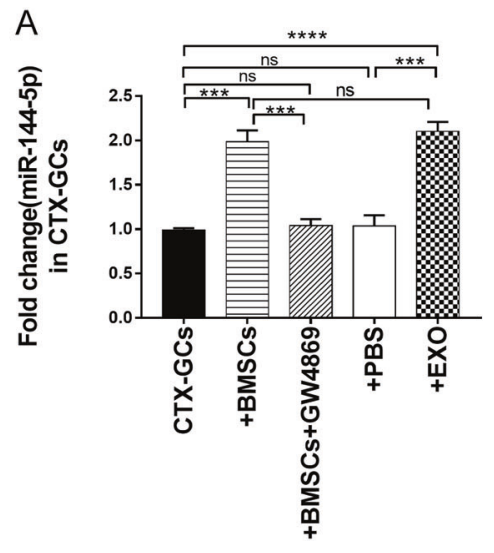

B

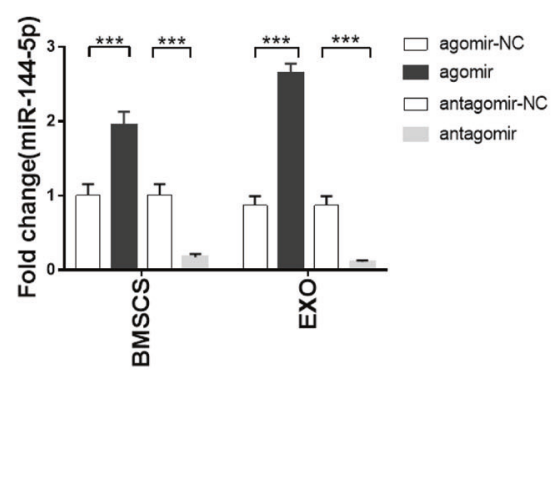

D
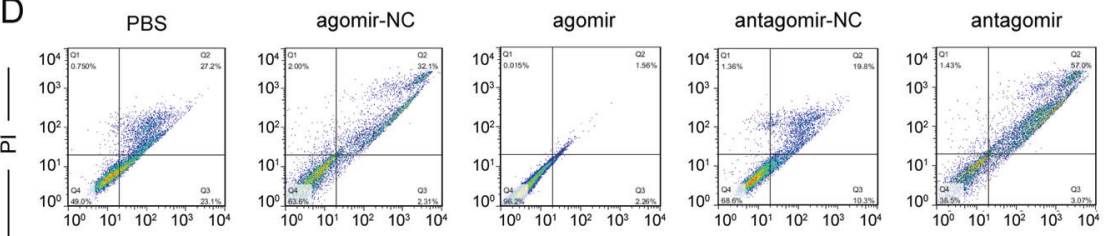

ANNEXIN-V FITC
$\mathrm{C}$

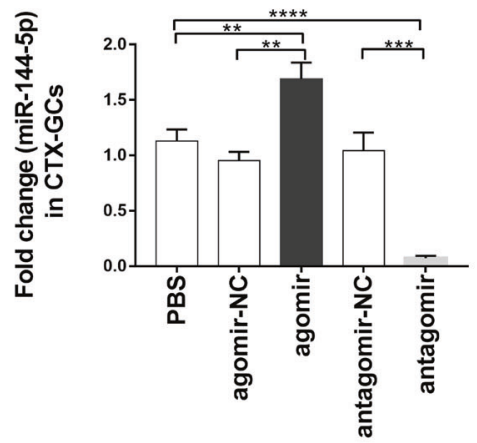

$\mathrm{E}$

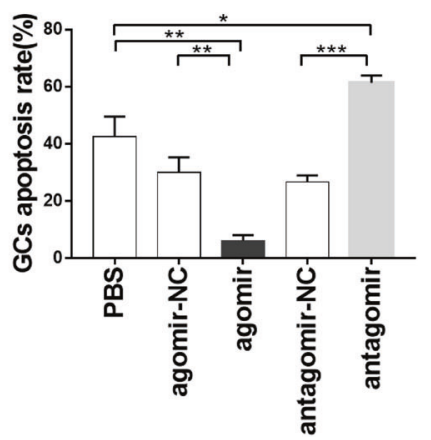

Fig. 4 MiR-144-5p improved the effects of BMSC-derived exosomes on damaged GCs. a The miR-144-5p expression in each group was detected by qRT-PCR. b miR-144-5p expression in BMSCs and exosomes after transfection. $\mathbf{c}$ The miR-144-5p expression levels in

pathway by the downstream target gene PTEN. To evaluate the association between BMSC exosomal miR-144-5p/ PTEN and the PI3K/AKT pathway in GCs, we assessed PTEN and p-AKT expression in CTX-damaged GCs cocultured with exosomes when overexpressing and inhibiting miR-144-5p. Both p-AKT upregulation and low PTEN expression were observed in GCs cocultured with exosomes that were pretreated with agomir. In contrast, both p-AKT downregulation and high PTEN expression were observed in GCs cocultured with exosomes that were pretreated with antagomir (Fig. 5d, e), which indicated that BMSC exosome miR-144-5p activated the PI3K/AKT pathway by PTEN targeting to inhibit the apoptosis of CTX-damaged GCs.

\section{Discussion}

POF, which occurs in 1\% of women aged 30-39 years, has a profound impact on the physical and mental health of females and has become one of the main causes of female infertility [30]. There is currently no effective measure to prevent or treat POF [31]. Follicle atresia, an important characteristic of $\mathrm{POF}$, is predominantly mediated by the apoptosis of follicular cells, especially GCs,
CTX-damaged GCs cocultured with BMSC-derived exosomes after transfection. d The apoptosis rate of GCs as determined by flow cytometry. e Comparison of the percentages of apoptotic GCs

which are essential to sustain follicle cell survival. Therefore, improving ovarian function and restoring the fertility of patients by suppressing GC apoptosis is very important.

Mesenchymal stem cells (MSCs) are known for their self-renewal and multilineage differentiation potential as well as for their prospective applications in cellular and genetic POF therapies. The efficacy of transplanting various types of MSCs, including human placenta mesenchymal stem cells [32], chorionic plates [33], and amniotic fluid stem cells [34], has been verified. However, the mechanisms underlying tissue repair have not been fully elucidated, and there is no evidence that transplanted MSCs directly differentiate into GCs to replace ovaries damaged by POF. The favorable effects of MSCs on CTX-damaged ovaries are thought to be due to the secretion of certain MSCderived beneficial factors that support ovarian follicular cell survival. A previous study confirmed that the growth factors secreted by MSCs, such as VEGF, IGF-1, and HGF, play important roles in restoring ovarian function [35]. As intercellular communication vehicles, exosomes might be involved in the repair process by preventing GC apoptosis [36, 37].

In our study, after the successful establishment of a POF rat model, BMSCs or BMSC-derived exosomes were 
A

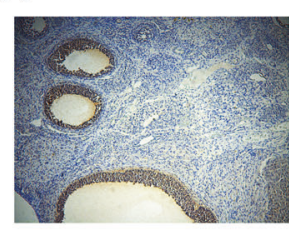

POF

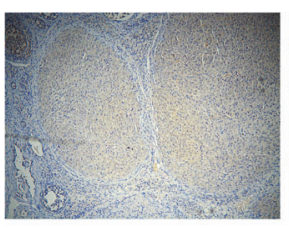

PBS

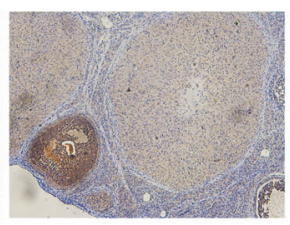

BMSCs

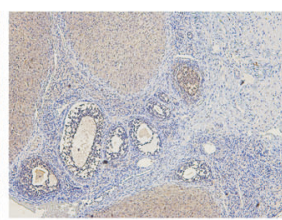

EXO

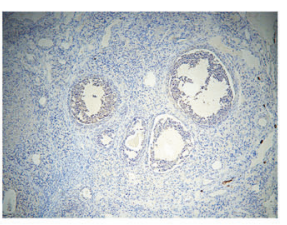

B

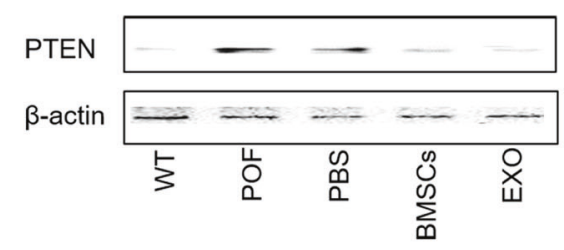

D

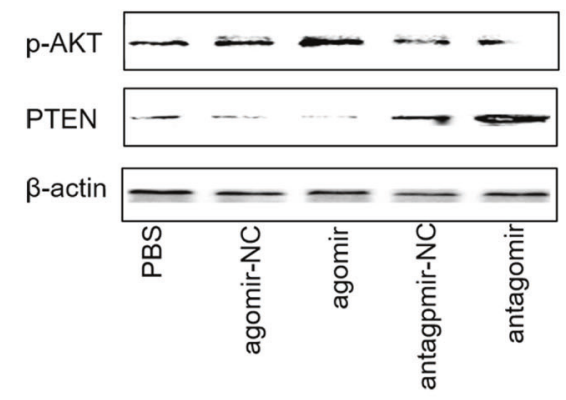

Fig. 5 Protein expression of PTEN and p-AKT. A-B PTEN in ovarian tissue was determined by immunohistochemistry (a) and western blot (b) analyses. c PTEN expression in CTX-damaged GCs cocultured with BMSCs, BMSCs pretreated with GW4869, PBS, or BMSC-

separately administered via intraperitoneal injection for treatment in vivo, and changes in ovarian function were detected by histopathological, serological, and cytological analyses. BMSC and BMSC-derived exosome transplantation increased the number of healthy follicles, restored the estrous cycle, and improved ovarian function, which is consistent with the findings of previous research [38]. In vitro, the apoptosis of CTX-damaged GCs was significantly inhibited after coculture with BMSCs or exosomes. However, when the exosomal release of BMSCs was prevented, the ability of BMSCs to inhibit GC apoptosis was weakened. This evidence suggested that BMSCs could transmit exosomes to inhibit the apoptosis of GCs and improve ovarian function.

The exosomal delivery of miRNAs is regarded as a novel and important mechanism of genetic exchange between cells [39]. Over the past several years, several studies have highlighted the potential therapeutic advantages of using exosomal miRNAs from MSCs for the treatment of various diseases and injuries [40-42].
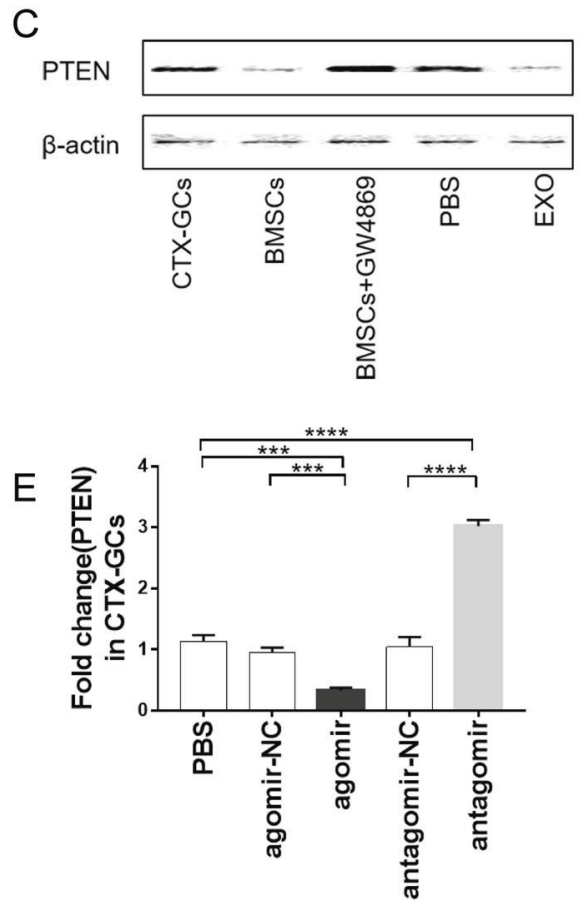

derived exosomes. $\mathbf{d}$ PTEN and p-AKT expression in CTX-damaged GCs cocultured with BMSC-derived exosomes after transfection. e PTEN expression was determined by qRT-PCR

In addition, we confirmed that the overexpression of miR-144-5p in BMSC-derived exosomes improved the therapeutic effects on CTX-damaged GCs. Knocking down miR-144-5p led to the promotion of CTX-damaged GC apoptosis, which was in agreement with previous studies revealing that miR-144-5p protected against POF in animal models. miR-144-5p expression can reportedly be used as a prognostic biomarker for esophageal carcinoma [43], gastric cancer [44], and breast cancer [45]. Contrary to our findings, a previous study reported that downregulation of miR-144-3p and miR-144-5p was frequently observed in bladder cancer cells and that miR-144-5p restoration inhibited cancer cell proliferation by inducing cell cycle arrest [46]. These miRNAs play complicated roles in a variety of physiological processes to maintain bodily homeostasis [47].

Furthermore, exosomal miR-144-5p in BMSCs was able to directly target PTEN, a protein involved in the apoptosis of CTX-damaged GCs. PTEN has been shown to negatively regulate the PI3K/AKT pathway and induce apoptosis or 
cell cycle arrest at the G1 phase [48]. In our experiments, miR-144-5p-mediated PTEN inhibition led to increased PI3K/AKT signal activation, suggesting that PI3K/AKT may be involved in the miR-144-5p/PTEN pathway.

Our findings imply that the delivery of BMSC-derived exosomal miR-144-5p could be beneficial for the preservation of ovarian follicles after chemotherapy-induced ovarian failure through the PTEN/PI3K/AKT axis. This study reveals that the delivery of exosomal miRNA is a critical part of stem cell-based POF therapy and avoids the disadvantages of direct MSC transplantation. Nevertheless, in terms of clinical practice, numerous problems remain to be addressed. Because the complex functions of BMSCderived exosomal miR-144-5p have not been determined, how to apply this approach in the treatment of POF in a targeted and quantitative way to avoid side effects still needs to be investigated. However, our study elucidates the potential clinical application value of exosomal miRNAmediated ovarian function recovery.

Acknowledgements This research was supported by the National Natural Science Foundation of China (grant nos. 81402165/81672913/ 81871343), the Social Development Project of Jiangsu (grant no. BE2018693/BE2017698), the Natural Science Foundation of Jiangsu Province (BK20171311/BK20181226), the Six Talent Peaks Project in Jiangsu Province (grant no. 2016-WSW-125), the "333 Project" Research Projects of Jiangsu Province (BRA2016141), the Jiangsu Provincial Medical Youth Talent (QNRC2016460, FRC201788), and the Scientific Research Project of Maternal and Child Health of Jiangsu Province (F201865)

\section{Compliance with ethical standards}

Conflict of interest The authors declare that they have no conflict of interest.

Publisher's note Springer Nature remains neutral with regard to jurisdictional claims in published maps and institutional affiliations.

\section{References}

1. Deepti G. Premature ovarian failure. Horm Res. 2007;68: 196-202.

2. Mahbod E. Pathogenesis and causes of premature ovarian failure: an update. Int J Fertil Steril. 2011;5:54-65.

3. Mousavi-Jarrrahi SH, Kasaeian A, Mansori K, Ranjbaran M, Khodadost M, Mosavi-Jarrahi A. Addressing the younger age at onset in breast cancer patients in Asia: an age-periodcohort analysis of fifty years of quality data from the international agency for research on cancer. ISRN Oncol. 2013; 2013:429862.

4. Gradishar WJ, Schilsky RL. Effects of cancer treatment on the reproductive system. Crit Rev Oncol/Hematol. 1988;8:153-71.

5. N S. Mechanisms of premature ovarian failure. Ann d'Endocrinol. 2003;64:87-92.

6. Akogullari D, Uluer ET, Vatansever HS. Investigation of the relation between follicular atresia and granulosa cells in terms of cell death mechanisms in premature ovarian failure model. Proceedings. 2018;2:1529.
7. Yu Bin D. Intravenously administered BMSCs reduce neuronal apoptosis and promote neuronal proliferation through the release of VEGF after stroke in rats. Neurol Res. 2010;32: $148-56$.

8. Hao L. Transplantation of BMSCs expressing hPDGF-A/hBD2 promotes wound healing in rats with combined radiation-wound injury. Gene Ther. 2009;16:34-42.

9. Enca M-R. Autologous bone marrow stem cells to treat acute myocardial infarction: a systematic review. Eur Heart J. 2008; 29:1807-18.

10. Hua-Lian H. Role of BMSCs in liver regeneration and metastasis after hepatectomy. World J Gastroenterol. 2014;20:126-32.

11. Ho-Joon L. Bone marrow transplantation generates immature oocytes and rescues long-term fertility in a preclinical mouse model of chemotherapy-induced premature ovarian failure. J Clin Oncol. 2007;25:3198-204.

12. Liu J, Zhang H, Zhang Y, Li N, Wen Y, Cao F, et al. Homing and restorative effects of bone marrow-derived mesenchymal stem cells on cisplatin injured ovaries in rats. Mol Cells. 2014;37:865-72.

13. Chen X, Wang Q, Li X, Wang Q, Xie J, Fu X. Heat shock pretreatment of mesenchymal stem cells for inhibiting the apoptosis of ovarian granulosa cells enhanced the repair effect on chemotherapy-induced premature ovarian failure. Stem Cell Res Ther. 2018;1:240.

14. A I C. Why are MSCs therapeutic? New data: new insight. J Pathol. 2009;2017:318-24.

15. James A. Mesenchymal stem cell therapy: two steps forward, one step back. Trends Mol Med. 2010;16:203-9.

16. Clotilde T. Exosomes: composition, biogenesis and function. Nat Rev Immunol. 2002;2:569-79.

17. Marote A, Teixeira FG, Mendes-Pinheiro B, Salgado AJ. MSCsderived exosomes: cell-secreted nanovesicles with regenerative potential. Front Pharmacol. 2016;7:231.

18. Ohno S, Kuroda M. Exosome-mediated targeted delivery of miRNAs. Methods Mol Biol. 2016;1448:261-70.

19. Zhang J, Li S, Li L, Li M, Guo C, Yao J, et al. Exosome and exosomal MicroRNA: trafficking, sorting, and function. Genom, Proteom Bioinform. 2015;13:17-24.

20. Guo Y, Sun J, Lai D. Role of microRNAs in premature ovarian insufficiency. Reprod Biol Endocrinol. 2017;15:38.

21. Yang X, Zhou Y, Peng S, Wu L, Lin HY, Wang S, et al. Differentially expressed plasma microRNAs in premature ovarian failure patients and the potential regulatory function of mir-23a in granulosa cell apoptosis. Reproduction. 2012;144:235-44.

22. Yujie D. MicroRNA-22-3p is down-regulated in the plasma of Han Chinese patients with premature ovarian failure. Fertil Steril. 2015;103:802-7 e1.

23. Haixue K. Profiling of differentially expressed microRNAs in premature ovarian failure in an animal model. Gynecol Endocrinol. 2014;30:57-61.

24. Zhu L, Li J, Xing N, Han D, Kuang H, Ge P. American ginseng regulates gene expression to protect against premature ovarian failure in rats. BioMed Res Int. 2015;2015:767124.

25. Liu T, Wang S, Li Q, Huang Y, Chen C, Zheng J. Telocytes as potential targets in a cyclophosphamide-induced animal model of premature ovarian failure. Mol Med Rep. 2016;3:2415-22.

26. Marcondes FK, Bianchi FJ, Tanno AP. Determination of the estrous cycle phases of rats: some helpful considerations. Braz J Biol. 2002;62:609-14.

27. Hirshfield AN. Development of follicles in the mammalian ovary. Int Rev Cytol. 1991;124:43-101.

28. Remmele W, Stegner HE. [Recommendation for uniform definition of an immunoreactive score (IRS) for immunohistochemical estrogen receptor detection (ER-ICA) in breast cancer tissue]. Der Pathologe. 1987;8:138-40. 
29. Zomer A, Vendrig T, Hopmans ES, van Eijndhoven M, Middeldorp JM, Pegtel DM. Exosomes: fit to deliver small RNA. Commun Integr Biol. 2010;3:447-50.

30. Benetti-Pinto CL, de Almeida DM, Makuch MY. Quality of life in women with premature ovarian failure. Gynecol Endocrinol. 2011;27:645-9.

31. Ertug K. Premature ovarian failure: clinical presentation and treatment. Obstet Gynecol Clin North Am. 2015;42:153-61.

32. Zhang H, Luo Q, Lu X, Yin N, Zhou D, Zhang L, et al. Effects of hPMSCs on granulosa cell apoptosis and AMH expression and their role in the restoration of ovary function in premature ovarian failure mice. Stem Cell Res Ther. 2018;9:20.

33. Li J, Yu Q, Huang H, Deng W, Cao X, Adu-Frimpong M, et al. Human chorionic plate-derived mesenchymal stem cells transplantation restores ovarian function in a chemotherapy-induced mouse model of premature ovarian failure. Stem Cell Res Ther. 2018;9:81.

34. Xiao GY, Liu IH, Cheng CC, Chang CC, Lee YH, Cheng WT, et al. Amniotic fluid stem cells prevent follicle atresia and rescue fertility of mice with premature ovarian failure induced by chemotherapy. PloS One. 2014;9:e106538.

35. Min S. Adipose-derived stem cells improved mouse ovary function after chemotherapy-induced ovary failure. Stem Cell Res Ther. 2013;4:80.

36. Xiao GY, Cheng CC, Chiang YS, Cheng WT, Liu IH, Wu SC. Exosomal miR-10a derived from amniotic fluid stem cells preserves ovarian follicles after chemotherapy. Sci Rep. 2016; 6:23120

37. Huang B, Lu J, Ding C, Zou Q, Wang W, Li H. Exosomes derived from human adipose mesenchymal stem cells improve ovary function of premature ovarian insufficiency by targeting SMAD. Stem Cell Res Ther. 2018;9:216.

38. Abd-Allah SH, Shalaby SM, Pasha HF, El-Shal AS, Raafat N, Shabrawy SM, et al. Mechanistic action of mesenchymal stem cell injection in the treatment of chemically induced ovarian failure in rabbits. Cytotherapy. 2013;15:64-75.
39. Valadi H, Ekstrom K, Bossios A, Sjostrand M, Lee JJ, Lotvall JO. Exosome-mediated transfer of mRNAs and microRNAs is a novel mechanism of genetic exchange between cells. Nat Cell Biol. 2007;9:654-9.

40. Shen H, Yao X, Li H, Li X, Zhang T, Sun Q. Role of exosomes derived from miR-133b modified MSCs in an experimental rat model of intracerebral hemorrhage. J Mol Neurosci. 2018;64:421-30.

41. Xiao C, Wang K, Xu Y, Hu H, Zhang N, Wang Y, et al. Transplanted mesenchymal stem cells reduce autophagic flux in infarcted hearts via the exosomal transfer of miR-125b. Circ Res. 2018;123:564-78.

42. Shi B, Wang Y, Zhao R, Long X, Deng W, Wang Z. Bone marrow mesenchymal stem cell-derived exosomal miR-21 protects C-kit+ cardiac stem cells from oxidative injury through the PTEN/PI3K/Akt axis. PloS One. 2018;13:e0191616.

43. Gao Z, Liu R, Liao J, Yang M, Pan E, Yin L, et al. Possible tumor suppressive role of the miR-144/451 cluster in esophageal carcinoma as determined by principal component regression analysis. Mol Med Rep. 2016;14:3805-13.

44. Wang Z, Wang Y, Yang T, Li J, Yang X. Erratum to: Study of the reparative effects of menstrual-derived stem cells on premature ovarian failure in mice. Stem Cell Res Ther. 2017;8:49.

45. Chin-Wen C. microRNA expression in prospectively collected blood as a potential biomarker of breast cancer risk in the BCFR. Anticancer Res. 2015;35:3969-77.

46. Matsushita R, Seki N, Chiyomaru T, Inoguchi S, Ishihara T, Goto $\mathrm{Y}$, et al. Tumour-suppressive microRNA-144-5p directly targets CCNE1/2 as potential prognostic markers in bladder cancer. Br J Cancer. 2015;113:282-9.

47. Schickel R. MicroRNAs: key players in the immune system, differentiation, tumorigenesis and cell death. Oncogene. 2008; 27:5959-74.

48. Zheng X. The inducible expression of the tumor suppressor gene PTEN promotes apoptosis and decreases cell size by inhibiting the PI3K/Akt pathway in Jurkat T cells. Cell Growth Differ. 2002; 13:285-96. 